\title{
Low risk of bacteremia after endoscopic variceal therapy for esophageal varices: a systematic review and meta-analysis
}

Authors

Institutions
Yi Jia ${ }^{1}$, Alok Dwivedi ${ }^{2}$, Sherif Elhanafi ${ }^{1}$, Arleen Ortiz ${ }^{1}$, Mohamed Othman ${ }^{1}$, Marc Zuckerman ${ }^{1}$

${ }^{1}$ Division of Gastroenterology, Texas Tech University Health Sciences Center, El Paso, Texas, USA

2 Division of Biostatistics \& Epidemiology, Texas Tech University Health Sciences Center, El Paso, Texas, USA submitted

29. December 2014 accepted after revision 7. May 2015

\section{Bibliography}

DOI http://dx.doi.org/

10.1055/s-0034-1392552

Published online: 11.8.2015

Endoscopy International Open

(c) Georg Thieme Verlag KG

Stuttgart · New York

E-ISSN 2196-9736

\section{Corresponding author}

\section{Marc J. Zuckerman, MD}

Texas Tech University Health

Sciences Center

4800 Alberta Avenue

El Paso

Texas 79905

USA

Fax: +1-915-545-6634

Marc.Zuckerman@ttuhsc.edu
2015; 03: E409-E417

Division of Gastroenterology

Background and study aims: Endoscopic variceal ligation (EVL) and endoscopic variceal sclerotherapy (EVS) are the main therapeutic procedures for the emergency treatment and secondary prophylaxis of esophageal varices in cirrhotics. Postendoscopic bacteremia has been reported after EVS and EVL, but data on the frequency of bacteremia are conflicting. This study aims to provide incidences of bacteremia after EVS and EVL in different settings through meta-analysis.

Methods: Only prospective or randomized studies were included in this meta-analysis. Binomial distribution was used to compute variance for each study. Random effects models were used as the final model for estimating the effect size and 95\% confidence interval. Adjusted effects were obtained using meta-regression analysis.

Results: Nineteen prospective studies involving 1001 procedures in 587 patients were included

\section{Introduction}

$\nabla$

Endoscopic treatment is the most reliable method for both therapy and secondary prophylaxis of esophageal variceal bleeding [1]. As with any endoscopic procedure, bacteremia can occur as a result of bacterial translocation of endogenous microbial flora into the bloodstream [2,3]. For instance, the incidence of bacteremia has been reported to be $4.2 \%$ after diagnostic esophagogastroduodenoscopy $[4,5]$. Given the relatively low incidence of bacteremia in endoscopic procedures, current guidelines do not suggest antibiotic prophylaxis for infective endocarditis [6]. Endoscopic variceal ligation (EVL) has replaced endoscopic variceal sclerotherapy (EVS) as an alternative and superior treatment for esophageal varices [6]. Although both procedures have recorded complications of infection, the incidence of transient bacteremia after $\operatorname{EVL}(3-6 \%)[7,8]$ has been suggested to be lower than that after EVS $(0-53 \%)[9-11]$. Clinically significant bacter- in the meta-analysis on the risk of bacteremia after EVS or EVL in cirrhotics with esophageal varices. The frequency of bacteremia after endoscopic variceal therapy was $13 \%$. The frequency of bacteremia after EVS (17\%) was higher than after EVL (6\%) with no statistically significant difference $(P=0.106)$. The frequency of bacteremia after elective EVS (14\%) was significantly less than after emergency EVS $(22 \%)(P<0.001)$. The frequency of bacteremia after elective $\operatorname{EVL}(7.6 \%)$ was not significantly different from after emergency EVL (3.2\%) $(P=0.850)$.

Conclusions: The incidence of bacteremia is low in patients with cirrhosis and varices after esophageal variceal therapy. These results are consistent with our current guidelines that antibiotic prophylaxis before endoscopic variceal therapy is only necessary for bleeding patients.

emia is rare after endoscopic procedures. Most patients experience only mild dysphagia, throat soreness, chest discomfort, and rarely spontaneous bacterial peritonitis. Moreover, the total incidence of all types of infectious complications after EVL (1.8\%) may be lower than that for EVS (18\%) [9], and a retrospective study concluded that the rate of clinical bacterial peritonitis after EVL may also be lower [7]. However, the existing data are conflicting due to the limited patient population sizes and different types of controls [7-11].

In the current guidelines addressing the risk of bacteremia with endoscopic variceal therapy, we noted the limited data on bacteremia with EVL $[2,12-14]$. In this study, we carried out a meta-analysis and present a systematic review to compare the frequency of bacteremia after EVL and EVS. The incidence of bacteremia was evaluated in upper gastrointestinal bleeding in patients with liver disease using endoscopic procedures in the emergency and elective settings. 


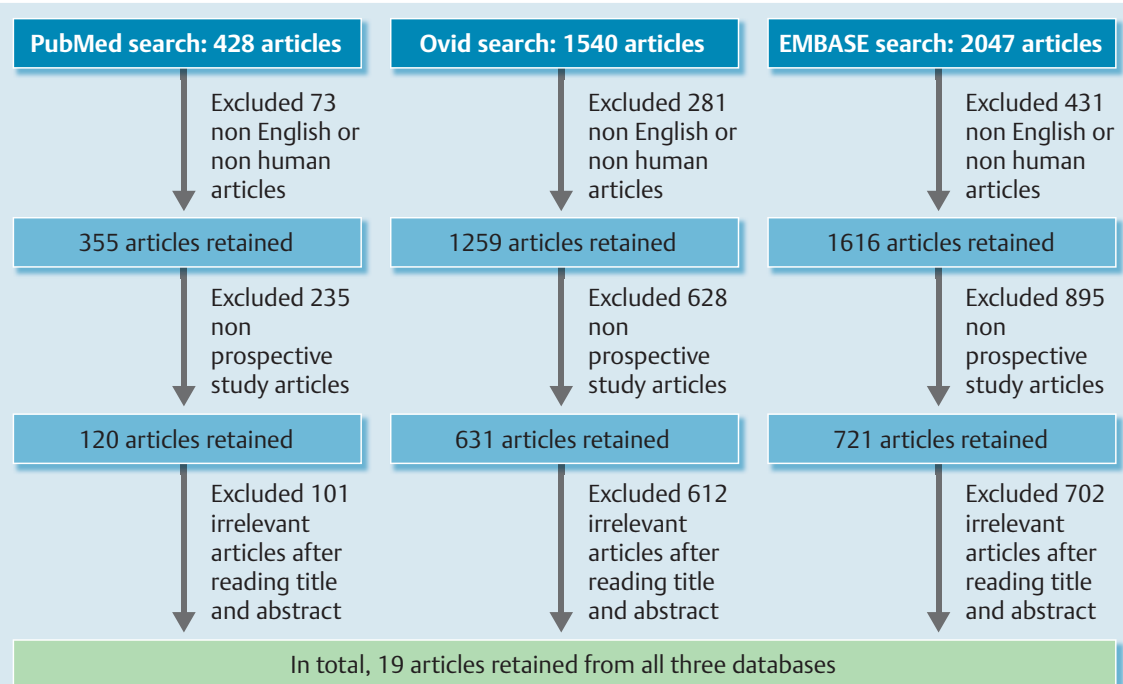

Methods

$\nabla$

\section{Materials and methods}

We followed the pre-specified and peer-reviewed PRISMA guidelines [15] for systematic review and meta-analyses statement, a 27-item checklist deemed essential for reporting of systematic reviews and meta-analysis of prospective studies.

\section{Search strategy}

We performed computerized searching along with manual searching of references of articles from digital dissertation databases, including PubMed, Ovid, and EMBASE from 1980 to August 2013. The search was limited to humans and English language papers. The search keywords and Boolean operators used were: esophageal varices, bacteremia, endoscopy, sclerotherapy OR banding OR ligation.

\section{Study selection}

Studies were eligible for inclusion in the meta-analysis if they met the following criteria: (1) they reported bacteremia in patients with cirrhosis and varices with either endoscopic sclerotherapy or ligation; (2) a Newcastle-Ottawa quality assessment score more than 7; (3) studies published in English language only. Case reports, reviews, articles published only in abstract form, or studies without the required data for meta-analysis were excluded from the analysis. Three reviewers (Y. J., S. E., and A. O.) performed the preliminary search independently using the above strategy and inclusion criteria to identify and access the primary articles with full text for inclusion in the pooled analysis. Data were independently extracted by these reviewers using a standardized extraction form and entered into an Excel 2010 (Microsoft, Redmond, WA, United States) spreadsheet. Information was collected with the data, including the patient population, etiology, positive culture and bacteremia rate, banding type, emergency or elective endoscopy setting, endoscopic sclerotherapy type, different sclerosant agents, and complications of the studies. Any differences in the collected data were resolved by discussing and reviewing the full text articles together among the reviewers.

\section{Newcastle-Ottawa quality assessment}

The quality of the paper was evaluated based on the NewcastleOttawa quality assessment [16]. The full text articles were reviewed independently and scores were provided by reviewers on selection, comparability, and outcome categories for each article. A total of 9 stars could be attributed overall. A score of 7 or higher was considered to be of good quality.

\section{Statistical analysis}

Random effects models were used to combine results of studies in the meta-analysis. Binomial distributions were used to compute variance for each study. The DerSimonian-Laird (DL) approach was used to determine the heterogeneity in the study. Heterogeneity was measured using I-squared. In this study, an Isquared value above $75 \%$ was considered to be indicative of a significant heterogeneity effect. In the presence of heterogeneity, a random effects model was considered to be the final model for estimating the effect size and $95 \%$ confidence interval $(\mathrm{CI})$. Heterogeneity was also explored using sub-group analysis. The risk of bacteremia between EVS and EVL studies was compared using univariate meta-regression analysis.

Unadjusted and adjusted random effect models were developed. The results of meta-regression were presented using the regression coefficient (RC) with $95 \% \mathrm{CI}$ and $P$ value. Some studies provided a comparison of bacteremia between elective and emergency settings. We used the relative risk (RR) measure to summarize such studies. Forest plots were constructed to summarize the studies. All of the statistical analyses were carried out using STAT 12.1. $P$ values less than $5 \%$ were considered to be significant results.

\section{Results \\ $\nabla$}

\section{Characteristics of selected studies}

The flow chart of the selected articles is shown in $\bullet$ Fig. 1. A total of 19 articles from the three digital dissertation databases were eligible for this study. Of these, four were randomized clinical trials [7,17-19], 12 were non-randomized studies with a control group $[8,11,20-29]$, and three were non-randomized studies without a control group $[4,9,10]$. According to Newcastle-Ottawa quality assessment, all of the included studies received more 
Table 1 Basic characteristics of the included studies with the quality scores based on the Newcastle-Ottawa quality assessment.

\begin{tabular}{|c|c|c|c|c|c|c|c|c|}
\hline \multirow[t]{3}{*}{ Author } & \multirow[t]{3}{*}{ Year } & \multirow[t]{3}{*}{ Country } & \multirow[t]{3}{*}{$\begin{array}{l}\text { Randomized } \\
(\mathrm{Y} / \mathrm{N})\end{array}$} & \multicolumn{4}{|c|}{$\begin{array}{l}\text { Rate of bacteremia } \\
\text { (+ culture/sessions) }\end{array}$} & \multirow[t]{3}{*}{$\begin{array}{l}\text { Quality } \\
\text { Score }\end{array}$} \\
\hline & & & & \multicolumn{2}{|l|}{ EVS } & \multicolumn{2}{|l|}{ EVL } & \\
\hline & & & & Emergency & Elective & Emergency & Elective & \\
\hline Cohen LB, et al & 1983 & USA & $\mathrm{N}$ & & $14 / 28$ & & & 7 \\
\hline Camara DS, et al & 1983 & USA & N & $2 / 40$ & & & & 7 \\
\hline Brayko CM, et al & 1985 & USA & N & $5 / 34$ & & & & 7 \\
\hline Snady H, et al & 1985 & USA & $\mathrm{N}$ & & $4 / 43$ & & & 7 \\
\hline Sauerbruch T, et al & 1985 & Germany & $\mathrm{N}$ & & $21 / 40$ & & & 7 \\
\hline Low DE, et al & 1986 & Canada & $\mathrm{N}$ & & $9 / 104$ & & & 7 \\
\hline Hegnhoj J, et al & 1988 & Denmark & N & & $7 / 31$ & & & 7 \\
\hline Lorgat F, et al & 1990 & South Africa & $\mathrm{N}$ & & $4 / 41$ & & & 7 \\
\hline $\mathrm{HoH}$, et al & 1991 & USA & N & $6 / 56$ & $0 / 33$ & & & 8 \\
\hline Tseng CC, et al & 1992 & USA & N & & & $0 / 3$ & $1 / 14$ & 7 \\
\hline Rolando N, et al & 1993 & UK & N & $46 / 115$ & $4 / 80$ & & & 8 \\
\hline Lo GH, et al & 1994 & Taiwan & Y & $10 / 58$ & & $2 / 60$ & & 8 \\
\hline Berner JS, et al & 1994 & USA & Y & & $1 / 9$ & & $0 / 11$ & 8 \\
\hline Selby WS, et al & 1994 & USA & N & & $7 / 20$ & & & 8 \\
\hline Rohr MRS, et al & 1997 & Brazil & $\mathrm{N}$ & & $2 / 43$ & & $2 / 35$ & 7 \\
\hline Kulkarni SG, et al & 1999 & India & Y & $6 / 8$ & $6 / 22$ & $0 / 2$ & $8 / 30$ & 8 \\
\hline Lin OS, et al & 2000 & Taiwan & N & & & & $11 / 67$ & 8 \\
\hline Manulaz, EB, et al & 2003 & Brazil & $\mathrm{N}$ & & & & $1 / 40$ & 8 \\
\hline Bonilha, DQ, et al & 2011 & Brazil & Y & & $0 / 72$ & & $3 / 65$ & 9 \\
\hline
\end{tabular}

EVS, endoscopic variceal sclerotherapy; EVL, endoscopic variceal ligation.

All non-randomized studies had a control group apart from Lin OS et al. (2001), Sauerbruch T et al. (1985), and Camara DS et al. (1983).

than 7 scores. Thus, a total of 19 studies involving 1001 procedures in 587 patients reporting the frequency of bacteremia post-endoscopy treatment in patients with cirrhosis and varices, and published between 1983 and 2011 were included in the meta-analysis ( $\bullet$ Fig. 1). The basic characteristics of the selected studies are included in Table 1 with quality scores based on the Newcastle-Ottawa quality assessment. All patients had portal hypertension, with the most common underlying etiology of cirrhosis being alcohol and viral infection.

Of the 19 included studies, 11 prospective studies described the rate of bacteremia after EVS, five studies provided the rate of bacteremia after EVS as well as after EVL, and three studies described the rate of bacteremia after only EVL. Thus, a total of 16 studies provided the rate of bacteremia after EVS and eight studies described the rate of bacteremia after EVL. Although current guidelines recommend antibiotic prophylaxis before endoscopy procedures for patients with active variceal bleeding [2,12-14,30], all of these studies included only patients who did not receive any antibiotics within 72 hours before EVS or EVL.

\section{Outcome of the meta-analysis}

\section{Combined}

Overall, the frequency of bacteremia was estimated to be $13 \%$ (95\%CI: 9-18\%) after endoscopic variceal sclerotherapy (EVS) or endoscopic variceal ligation (EVL) using a random-effects model ( Fig. 2). The frequency of bacteremia after EVS was 17\% (95\% $\mathrm{CI}$ : $11-24 \%)$, which was higher than the frequency of bacteremia after EVL, which was 6\% (95\%CI: $2-11 \%)$, but the difference was not statistically significant $(P=0.106)$. The incidence of bacteremia ranged from $0 \%$ to $52 \%$ with substantial heterogeneity among the estimates $\left(\mathrm{I}^{2}=89 \%, P<0.01\right)$ in EVS studies, while it ranged from $0 \%$ to $25 \%$ in EVL studies without heterogeneity among the estimates $\left(\mathrm{I}^{2}=45.5 \%, P=0.076\right)$.
To explore the heterogeneity effect, in the first sensitivity analysis, we only included the four randomized studies with both EVS and EVL ( $\bullet$ Fig. 3 a). The frequency of bacteremia after EVS was 16\% (95\%CI: $0-33 \%)$, after EVL it was 6\% (95\%CI: $0-13 \%)$, and combined it was $10 \%$ (95\% CI: 3-16\%). After excluding these four randomized studies and considering only the 15 observational studies ( $\bullet$ Fig. $\mathbf{3 b}$ ), these indicated similar results. The frequency of bacteremia after EVS was 18\% (95\%CI: $11-25 \%)$, after EVL it was 7\% (95\%CI: $1-14 \%)$, and combined it was $15 \%$ (95\%CI: $9-21 \%)$.

In the second sensitivity analysis, we compared the five studies conducted with both EVS and EVL ( $\bullet$ Fig.3c), which indicated similar results for frequency of bacteremia: EVS 12\% (95\%CI: 1 $23 \%$ ), EVL 6\% (95\%CI: $1-11 \%)$, combined 8\% (95\%CI: 3-13\%). After excluding these five studies ( $\bullet$ Fig.3 d), the risk of bacteremia after EVS was 19\% (95\%CI: $12-27 \%$ ), and after EVL it was $8 \%$ (95\% CI: $-1 \%$ to $17 \%$ ).

- Table 2a shows the comparison of risk of bacteremia between EVS and EVL. The overall mean difference in the risk of bacteremia between EVS and EVL was estimated to be $10 \%$, while it was $12 \%$ after excluding studies that compared EVS and EVL, and 11\% in nonrandomized studies. When we only included studies that compared EVS and EVL, we found a $63 \%$ increased risk of bacteremia in EVS compared with EVL and an $84 \%$ increased risk of bacteremia in EVS compared with EVL when we only included randomized studies $(P=0.293)$.

We also compared the incidence of bacteremia according to whether the setting was either an emergency or an elective procedure irrespective of whether they were EVS or EVL studies ( Fig.4a-c). A total of six studies had emergency EVS or EVL (two with both EVS and EVL) with a frequency of bacteremia of $16 \%$ (95\%CI: $5-27 \%$ ) and a total of 16 studies had elective EVS or EVL (four with both EVS and EVL) with a frequency of bacteremia of $12 \%$ (95\%CI: $7-16 \%$ ) ( Fig. 4 a, $\bullet$ Table 2 b). When EVS or 


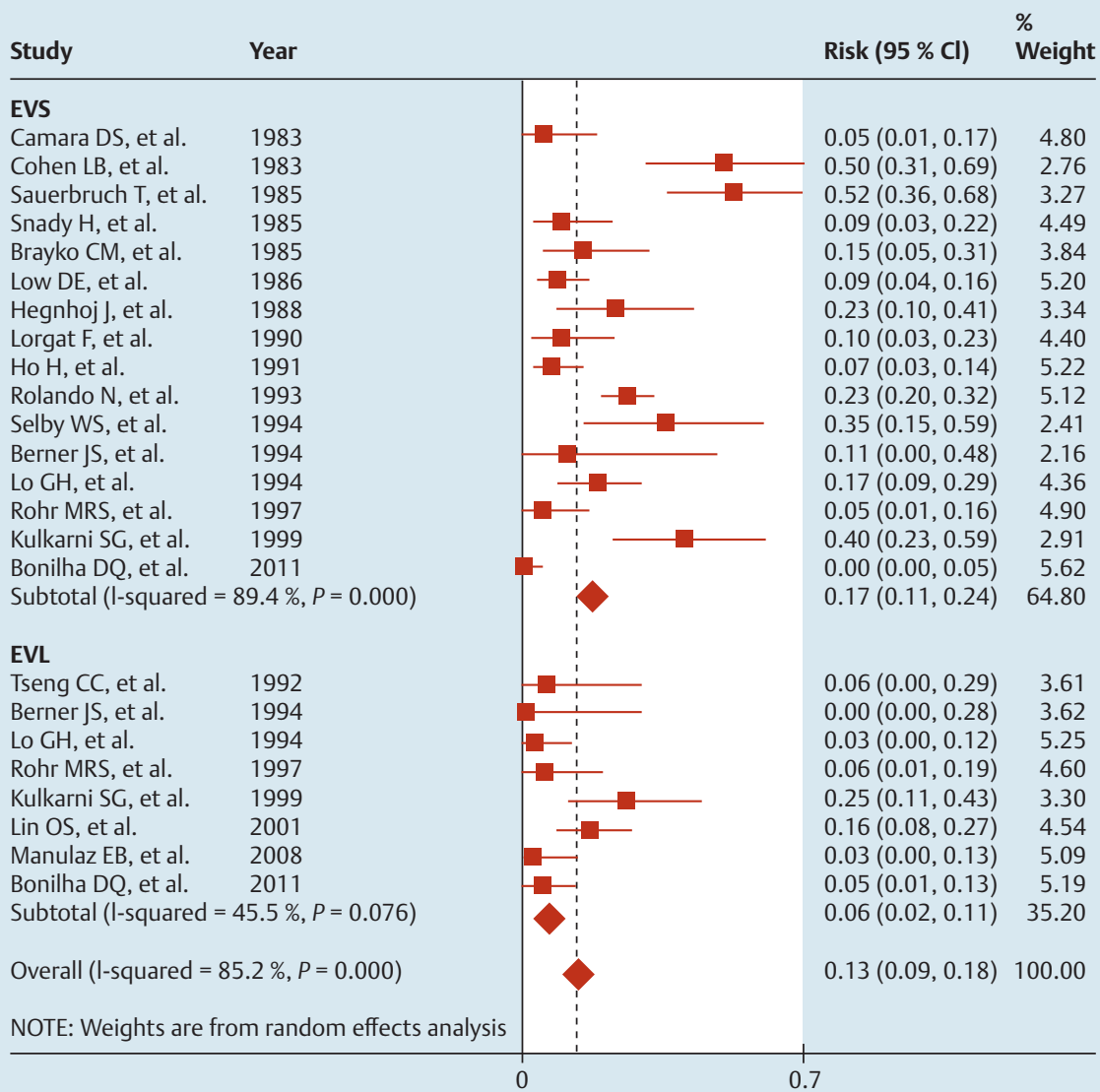

Fig. 2 Risk of bacteremia after endoscopic variceal sclerotherapy (EVS) and endoscopic variceal ligation (EVL).
EVL were performed as elective procedures, the risk of bacteremia after these procedures was $6 \%$ lower compared with emergency procedures. The pooled estimate from within studies comparisons showed that elective procedures have a $71 \%$ lower risk of bacteremia compared with emergency procedures and the difference was statistically significant $(P=0.006)$, while there was a $79 \%$ lower risk of bacteremia during elective procedures compared with emergency procedures within EVS studies, and again the difference was statistically significant $(P=0.002)$. In contrast, within EVL studies, elective procedures had a $21 \%$ higher risk of bacteremia compared to emergency procedures but the difference was not statistically significant $(P=0.850)$.

\section{EVS or EVL}

In total, 16 studies with 877 procedures in 463 patients (295 male/112 female/56 unspecified) with cirrhosis and varices provided data on the frequency of bacteremia after EVS. Elective EVS was done in 566 sessions in 13 studies with a frequency of bacteremia of $14.4 \%$ (95\%CI: $8-20.8 \%$ ) using a random-effects model, while emergency EVS was performed in 271 sessions in five studies with a frequency of bacteremia of $22.4 \%$ (95\% CI: $9.2-35.6 \%$ ) ( Supplementary Table 3 a). The pooled analysis of the three studies that conducted both emergency and elective EVS indicated a significantly lower frequency of bacteremia after elective EVS than emergency EVS. The relative risk of elective EVS versus emergency EVS was 0.21 (95\%CI: $0.077-0.572, P=0.002$ ) ( Fig. 4b, $\odot$ Supplementary Table 3b). Different sclerosants and differen sclerosant injection technique did not affect the frequency of bacteremia after EVS ( Supplementary Tables 3a-3b).

Eight studies with a total of 327 procedures in 249 patients (174 male/75 female) with cirrhosis and varices provided data on the frequency of bacteremia after EVL. Elective EVL was done in 262 sessions in seven studies with a frequency of bacteremia of $7.6 \%$ (95\%CI: $2.3-13 \%$ ) using a random-effects model, while emergency EVL was performed in 65 sessions in three studies with a frequency of bacteremia of $3.2 \%$ (95\%CI: $-2.2 \%$ to $8.6 \%$ ) ( Supplementary Table $4 \mathrm{a}$ ). The pooled analysis of the two studies conducting both emergency and elective EVL indicated no statistically significant difference between the frequency of bacteremia after elective EVL and after emergency EVL, and the relative risk of elective EVL to emergency EVL was 1.21 (95\%CI: 0.17-8.62, $P=$ 0.850) ( Fig. 4c, $\bigcirc$ Table 2c). Different banding techniques (single or multiple) did not affect the frequency of bacteremia after EVL $\odot$ Supplementary Tables $4 a-4 c)$.

\section{Organisms}

A total of 25 different organisms ( 24 after EVS, 7 after EVL) were reported in 160 blood cultures after EVL and EVS in the 19 studies ( Table5). The most common bacteria found in blood cultures after EVL and EVS was alpha-hemolytic Streptococcus ( $\mathrm{n}=$ 46) (mainly Streptococcus viridans) which was $28.8 \%$ of all reported organisms. The other common bacteria were coagulasenegative Staphylococcus (mainly Staphylococcus epidermidis) (n =39), followed by coagulase-positive Staphylococcus (mainly Staphylococcus aureus) $(\mathrm{n}=15)$. Gram-negative Escherichia coli and Klebsiella were less commonly reported than the Gram-positive bacteria.

\section{Complications}

No clinical evidence of infection occurred in patients in 15 of the 19 studies. Some patients did report mild post-procedure fever or leukocytosis, but no sources were identified and these symptoms may not be related to the procedures $[7,22,26]$. The most frequently encountered infectious complication in the other four 


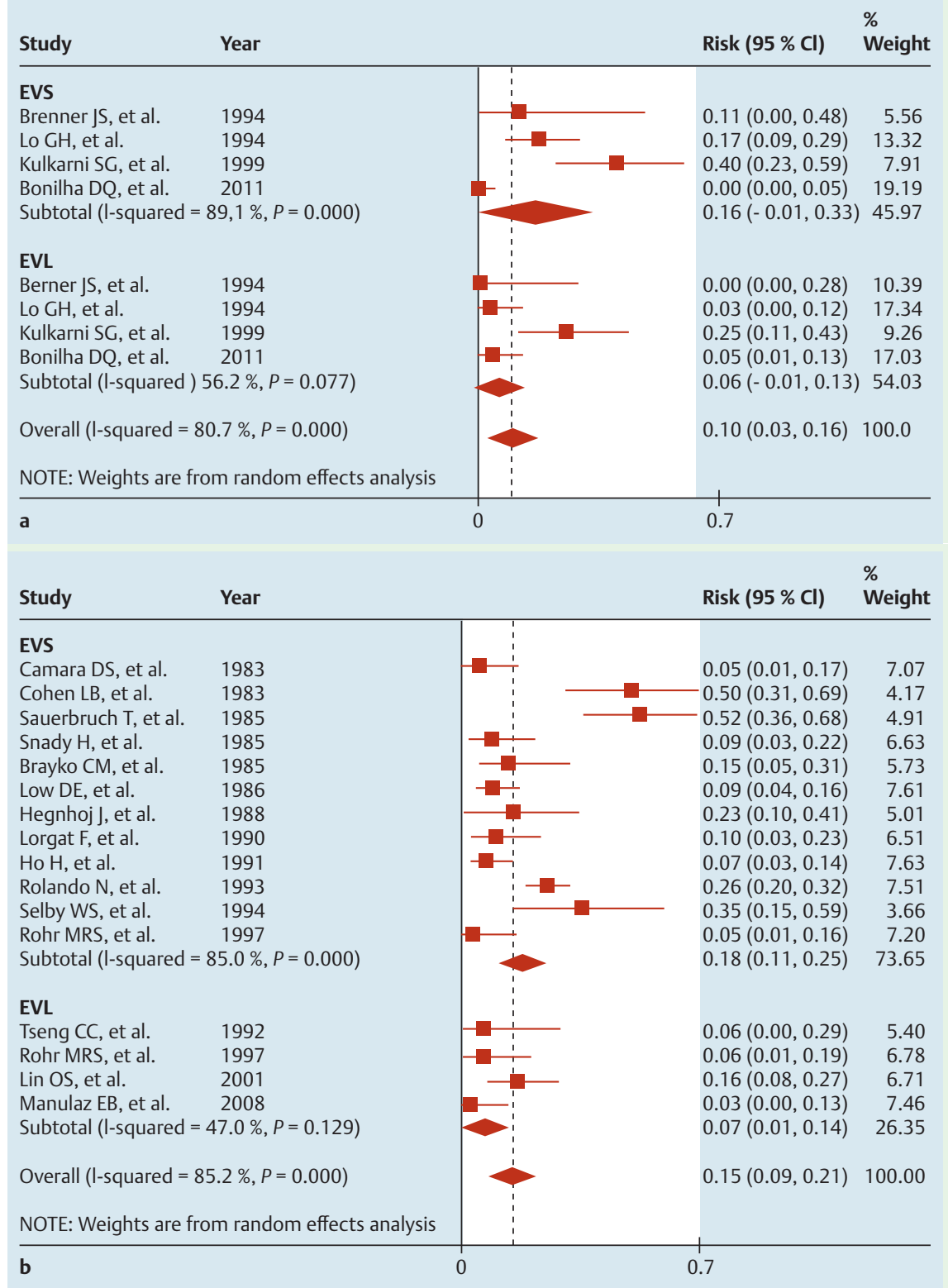

Fig. 3 Risk of bacteremia: sensitivity analysis. a Randomized studies that included both endoscopic variceal sclerotherapy (EVS) and endoscopic variceal ligation $(E V L)(n=4)$. b After EVS and EVL after excluding randomized studies $(n=15)$.

Continuation see following page studies was spontaneous bacterial peritonitis (SBP) in six cases $[7,18,26]$. All but one case of SBP occurred in patients with Child-Pugh class C cirrhosis [7]. One patient died of sepsis after EVS with injection of sodium tetradecyl [7]. Of note, no patient from these 19 studies had artificial heart valves.

\section{Discussion \\ $\nabla$}

We found the frequency of bacteremia after endoscopic variceal therapy to be $13 \%$. The frequency of bacteremia after EVS (17\%) was higher than after EVL (6\%) although the difference was not statistically significant. The frequency of bacteremia after elective EVS (13.1\%) was significantly lower than after emergency EVS (22.5\%), while the frequency of bacteremia after elective EVL (7.6\%) was not significantly different from that after emergency $\operatorname{EVL}(3.2 \%)$.

EVS is an effective therapy, carried out with intravariceal or paravariceal injection of sclerosant agents. EVL has replaced EVS as an alternative and superior treatment for esophageal varices with greater efficacy and lower complication rates [31 - 35]. In our eli- gible studies, most EVS and EVL procedures did not result in infectious complications, especially after EVL procedures for esophageal varices. The most frequent infectious complication was SBP, but this was uncommon $[7,18,26]$.

Both EVL and EVS have had reports of transient bacteremia, and the incidence of infectious complications after EVL was suspected to be lower than that after EVS, although the existing data are conflicting [7,17-19,28]. Early studies reported higher transient bacteremia in the EVS group than in the EVL group that was either statistically significant, $17.2 \%$ after EVS compared with $3.3 \%$ after EVL $(P<0.03)$ [7], or not statistically significant, $40 \%$ after EVS compared with 25\% after EVL [18]. Most recent studies found higher rates of positive blood cultures after EVL compared with after EVS (5.7\% after EVL compared with $4.6 \%$ after EVS [28], $4.6 \%$ after EVL compared with $0.0 \%$ after EVS [19]). Our metaregression analysis of the frequency of bacteremia after EVS was $17 \%$ with a trend toward being higher than after EVL (6\%). The risks of transient bacteremia are different in emergency and elective procedure settings $[11,18]$. Patients with active or recent bleeding may have variceal walls more susceptible to bacterial invasion. Our results indicate that the frequency of bacteremia after 


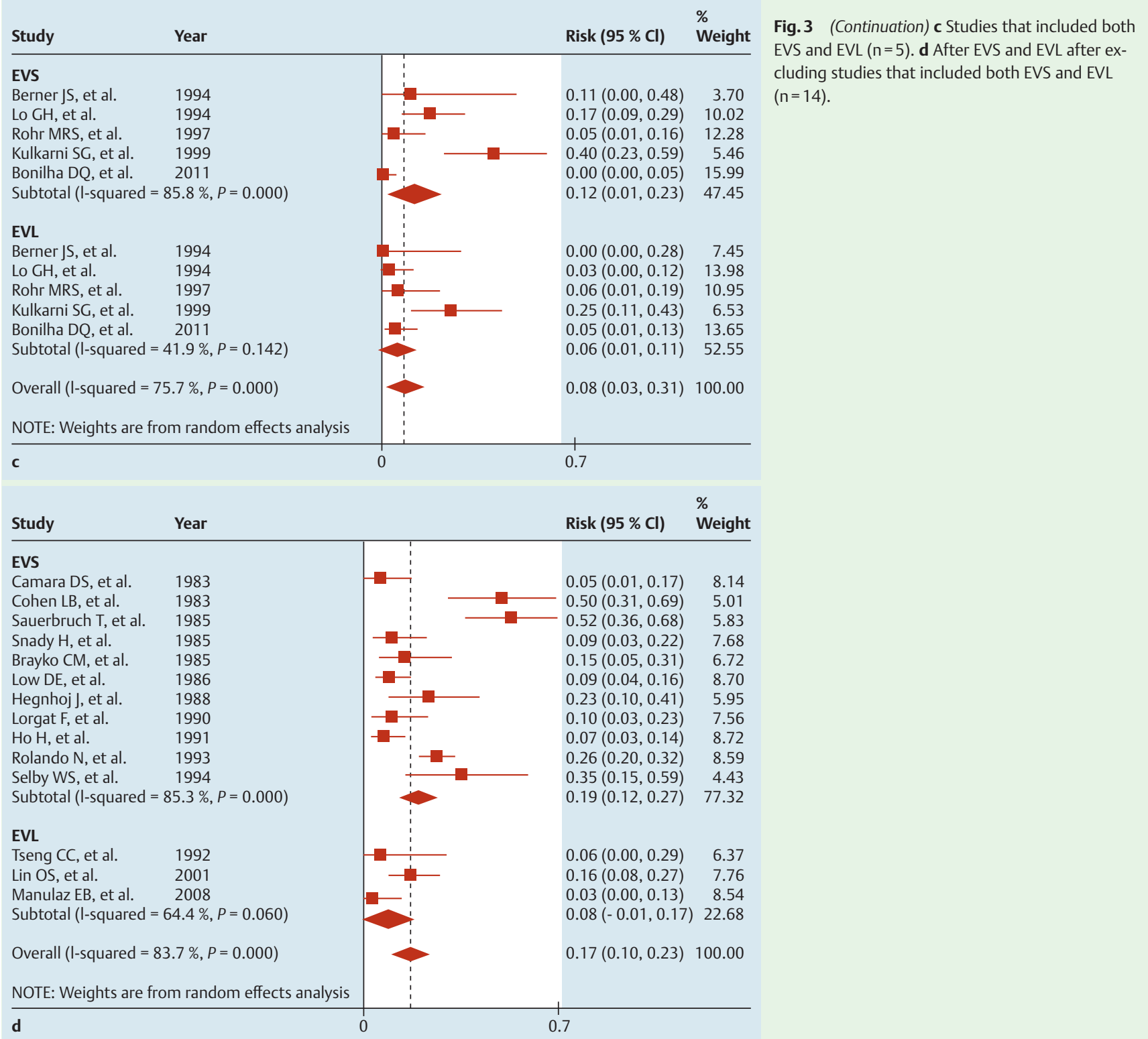

emergency EVS or EVL (16\%) is significantly higher than after elective EVS or EVL (12\%). The frequency of bacteremia after emergency EVS (22\%) is also significantly higher than after elective EVS (14\%).

Bacteremia may be due to oral or digestive pathogens from transient contamination [36], potential transluminal seeding from the needle and tips, contamination of the side channel of the endoscope, or contamination of sclerosant [37]. EVL does not involve the direct penetration of the esophageal mucosa with a needle and has less opportunity for the direct introduction of bacteria. Additionally, EVL using the single banding technique was done with a protective overtube that could prevent ligation bands from picking up oropharyngeal flora on the way in [7]. Furthermore, the process of ligation itself obliterates submucosal venous channels, reducing the likelihood of systemic bacteremia [8].

Many organisms reported to cause bacteremia after EVS and EVL were from skin and oropharyngeal sources, such as alpha-hemolytic Streptococcus, coagulase-negative Staphylococcus, and Diphtheroid species [11,13]. Coagulase-negative Staphylococcus is one of the most frequent causes of nosocomial bloodstream infection and sometimes occurs in patients with no clinically significant presentation [38]. These outside sources turned out to be the origin of infection because the same organism was grown from the endoscope $[39,40]$.

The other organisms isolated after EVS and EVL are coagulasepositive Staphylococcus and Gram-negative bacteria. After disruption of the intact mucosa member, Gram-negative bacteria can produce endotoxins and stimulate the release of tumor necrosis factor and bacterial translocation [41]. Endotoxemia could also induce nitric oxide synthase to produce vascular nitric oxide and increase the membrane permeability in the vascular endothelium and intestinal mucosa, possibly contributing to bacterial translocation [42]. The higher intestinal permeability index at the time of infection is a significant predictor for bacteremia [43]. Current guidelines recommend antibiotic prophylaxis before endoscopic procedures for patients with active variceal bleeding $[2,12-14,30]$. Meta-analysis studies indicated a significant decrease in the incidence of bacterial infections and mortality after antibiotic prophylaxis in cirrhotic patients with active gastrointestinal bleeding [2]. On the other hand, the guidelines do not specifically recommend antibiotic prophylaxis for patients un- 


\begin{tabular}{|c|c|c|c|c|c|}
\hline Type of study & $\mathbf{n}$ & RC & $95 \% \mathrm{Cl}$ & & $P$ value \\
\hline EVS with EVL as referent ${ }^{1}$ & 24 & 0.098 & -0.023 & 0.219 & 0.106 \\
\hline EVS with EVL as referent ${ }^{2}$ & 14 & 0.116 & -0.097 & 0.329 & 0.257 \\
\hline EVS with EVL as referent ${ }^{3}$ & 16 & 0.107 & -0.071 & 0.284 & 0.218 \\
\hline EVS with EVL as referent ${ }^{4}$ & 5 & $1.629^{5}$ & 0.656 & 4.047 & 0.293 \\
\hline EVS with EVL as referent ${ }^{1}$ (only randomized) ${ }^{6}$ & 4 & $1.837^{5}$ & 0.591 & 5.717 & 0.293 \\
\hline
\end{tabular}

Table 2 a Effect of endoscopic variceal sclerotherapy (EVS) on risk of bacteremia compared to endoscopic variceal ligation (EVL).

$\mathrm{RC}$, regression coefficient; $\mathrm{Cl}$, confidence interval.

${ }^{1}$ In all studies

2 after excluding studies which compared EVS and EVL

3 only in nonrandomized studies.

${ }^{4}$ Studies which compared EVS and EVL

${ }^{5}$ Relative risk

${ }^{6}$ randomized studies

\begin{tabular}{|lcllll|}
\hline Setting & $\mathbf{n}$ & I-squared & Risk & 95\%Cl & \\
\hline Elective & 20 & 0.817 & 0.116 & 0.071 & 0.162 \\
\hline Emergency & 8 & 0.801 & 0.161 & 0.048 & 0.274 \\
\hline
\end{tabular}

$\mathrm{Cl}$, confidence interval.

Table 2 b Overall risk of bacteremia after endoscopic variceal sclerotherapy (EVS) and endoscopic variceal ligation (EVL), comparison of emergency with elective procedures.

Table 2c

Comparison of elective versus emergency procedures.

\begin{tabular}{|c|c|c|c|c|c|}
\hline Setting & $\mathbf{n}$ & $\mathbf{R R}$ & $95 \% \mathrm{Cl}$ & & $P$ value \\
\hline Elective versus emergency as referent & 23 & $-0.064^{1}$ & -0.236 & 0.107 & 0.444 \\
\hline Elective versus emergency as referent (within studies comparisons) & 5 & 0.285 & 0.117 & 0.698 & 0.006 \\
\hline Elective versus emergency as referent (within studies comparisons) in EVS studies & 3 & 0.21 & 0.077 & 0.572 & 0.002 \\
\hline Elective versus emergency as referent (within studies comparisons) in EVL studies & 2 & 1.21 & 0.17 & 8.62 & 0.850 \\
\hline
\end{tabular}

$\mathrm{Cl}$, confidence interval; EVS, endoscopic variceal sclerotherapy; EVL, endoscopic variceal ligation; RR, relative risk.

1 Regression coefficient

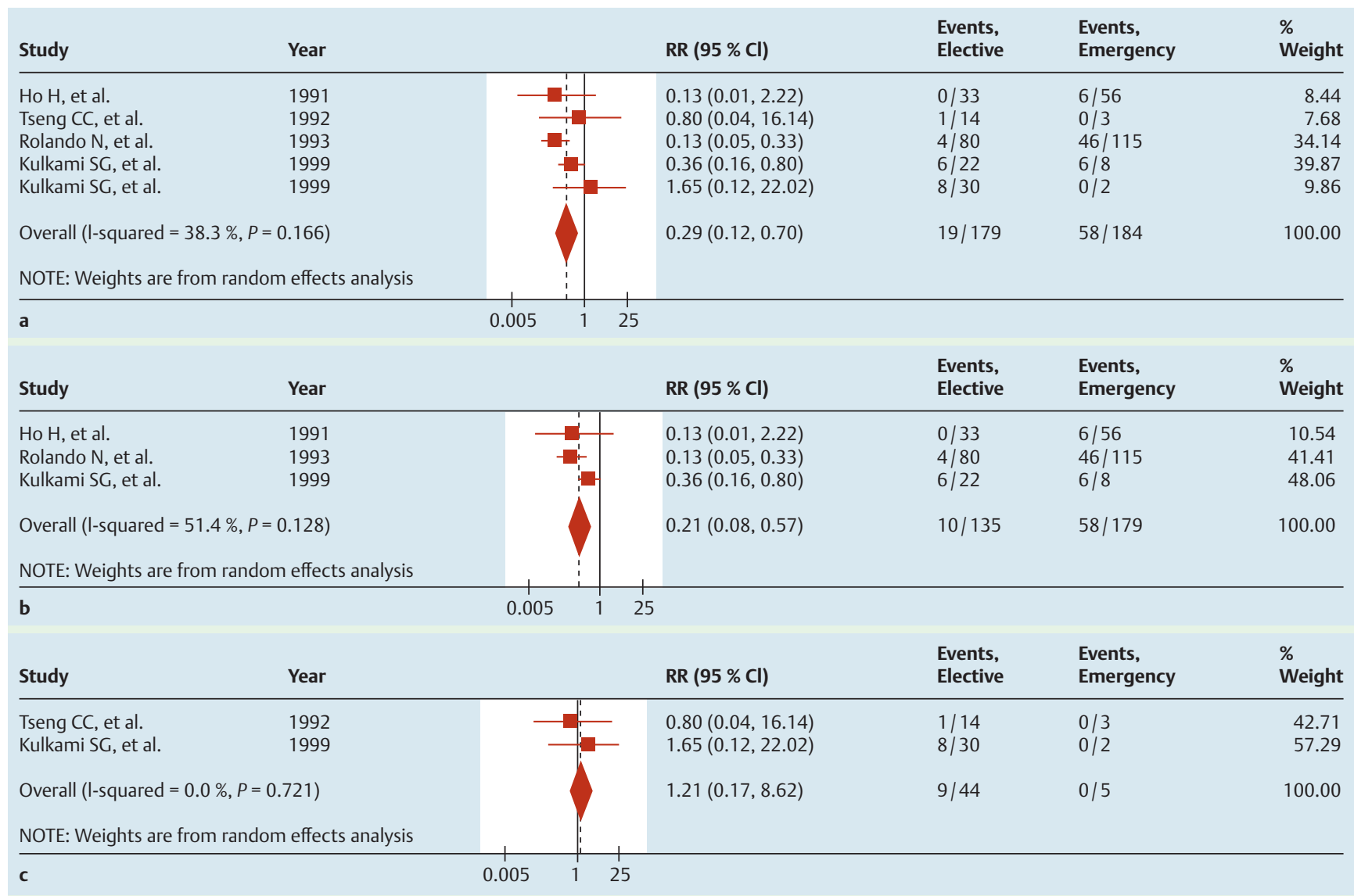

Fig. 4 Comparison of risk of bacteremia. a Between emergency and elective variceal therapy procedures (favors elective). b Between elective and emergency procedures in endoscopic variceal sclerotherapy (EVS) studies (favors elective). c Between elective and emergency procedures in endoscopic variceal ligation (EVL) studies (favors emergency). 
Table 5 Organisms from blood cultures.

\begin{tabular}{|lccc|}
\hline & EVS & EVL & Total \\
\hline Streptococcus, alpha-hemolytic & 40 & 6 & 46 \\
\hline Streptococcus, beta-hemolytic & & 3 & 3 \\
\hline Streptococcus, non-hemolytic & 3 & & 3 \\
\hline Staphylococcus, coagulase-negative & 28 & 11 & 39 \\
\hline Staphylococcus, coagulase-positive & 12 & 3 & 15 \\
\hline Escherichia coli & 6 & 1 & 7 \\
\hline Klebsiella spp. & 4 & 2 & 6 \\
\hline Pseudomonas spp. & 6 & & 6 \\
\hline Enterobacter cloacae & 3 & 2 & 5 \\
\hline Corynebacterium & 4 & & 4 \\
\hline Veillonella & 4 & & 4 \\
\hline Acinetobacter spp. & 3 & & 3 \\
\hline Diphtheroid & 3 & & 3 \\
\hline Bacillis spp. & 2 & & 2 \\
\hline Lactobacillus & 2 & & 2 \\
\hline Flavobacterium menigosepticum & 2 & & 2 \\
\hline Peptostreptococcus spp. & 2 & & 2 \\
\hline Clostridium spp. & 1 & & 1 \\
\hline Propionibacterium acnes & 1 & & 1 \\
\hline Alcaligenes faecalis & 1 & 1 \\
\hline Haemophilus influenza & 1 & 1 \\
\hline Bacterioides spp. & 1 & 1 \\
\hline Propionibacterium & 1 & 1 \\
\hline Xanthomonas maltophilia & 1 & 1 \\
\hline Neisseria spp. & 1 & 1 \\
\hline EVS, & & \\
\hline
\end{tabular}

EVS, endoscopic variceal sclerotherapy; EVL, endoscopic variceal ligation.

dergoing EVL or EVS for non-bleeding varices $[12,30]$. Since EVL is currently the standard procedure utilized for endoscopic variceal therapy, the main area of concern is whether antibiotic prophylaxis is needed before EVL in elective procedures. Prophylactic antibiotics should be individualized by different clinical presentations of patients instead of for the indication of EVL or EVS [12]. Currently, prophylactic antibiotics are indicated for all cirrhotic patients at high risk of developing infection, including patients with Child's class C cirrhosis, a recent history of variceal bleeding, a past history of bacterial peritonitis, or a co-morbid immunosuppression [30]. Our data indicate a low frequency of bacteremia after EVS and EVL, which is consistent with the current guideline recommendations.

There are some limitations to our analysis. First, many of the eligible studies in our systematic review do not distinguish between clinically significant and non-significant (possible contamination) bacteremia. We used all of the reported positive culture results in our meta-analysis. Second, the sample sizes in some comparisons are limited. These may cause the non-significant meta-analysis results. Third, most of the EVL studies used the older single banding technique rather than the current multi-banding ligators, which may affect the applicability of our results. Although our analysis included both EVS and EVL, EVS is not currently used as first-line therapy for esophageal varices. However, given that most of this literature is not recent, it forms the basis of our current guidelines.

In conclusion, the incidence of bacteremia is low in patients with cirrhosis and varices after EVL or EVS. The risk of bacteremia in patients with cirrhosis and varices is higher, but not significantly different, after EVS compared with EVL. The risk of bacteremia is significantly higher after emergency EVS than after elective EVS. These results are consistent with our current guidelines that antibiotic prophylaxis before endoscopic procedures is only neces- sary for patients with active variceal bleeding, but is not recommended for patients with non-bleeding varices. However, in view of even the low risk of bacteremia after EVL, it may be reasonable to give antibiotic prophylaxis to cirrhotics at high risk of infection, such as those with Child's class C cirrhosis and ascites.

\section{Competing interests: None}

\section{References}

1 Riggio O, Angeloni S, Nicolini G et al. Endoscopic screening for esophageal varices in cirrhotic patients. Hepatology 2002; 35: 501 - 502

2 Banerjee S, Shen B, Baron TH et al. Antibiotic prophylaxis for GI endoscopy. Gastrointest Endosc 2008; 67: 791 - 798

3 Zuckerman MJ, Menzies IS, Ho H et al. Assessment of intestinal permeability and absorption in cirrhotic patients with ascites using combined sugar probes. Dig Dis Sci 2004; 49: 621 - 626

4 Lin OS, Wu SS, Chen YY et al. Bacterial peritonitis after elective endoscopic variceal ligation: a prospective study. Am J Gastroenterol 2000; 95: $214-217$

5 Nelson $D B$. Infection control during gastrointestinal endoscopy. Can J Gastroenterol 2007; 21: 13-15

6 Brann OS. Infectious complications of cirrhosis. Curr Gastroenterol Rep 2001; 3: $285-292$

7 Lo GH, Lai KH, Shen MT et al. A comparison of the incidence of transient bacteremia and infectious sequelae after sclerotherapy and rubber band ligation of bleeding esophageal varices. Gastrointest Endosc 1994; 40: 675 - 679

8 Tseng CC, Green RM, Burke SK et al. Bacteremia after endoscopic band ligation of esophageal varices. Gastrointest Endosc 1992; 38: 336 - 337

9 Sauerbruch T, Holl J, Ruckdeschel G et al. Bacteriaemia associated with endoscopic sclerotherapy of oesophageal varices. Endoscopy 1985; 17: $170-172$

10 Camara DS, Gruber M, Barde CJ et al. Transient bacteremia following endoscopic injection sclerotherapy of esophageal varices. Arch Intern Med 1983; 143: 1350-1352

11 Ho H, Zuckerman MJ, Wassem C. A prospective controlled study of the risk of bacteremia in emergency sclerotherapy of esophageal varices. Gastroenterology 1991; 101: 1642 - 1648

12 Qureshi W, Adler DG, Davila R et al. ASGE Guideline: the role of endoscopy in the management of variceal hemorrhage, updated July 2005. Gastrointest Endosc 2005; 62: 651 - 655

13 Garcia-Tsao G, Sanyal AJ, Grace ND et al. Prevention and management of gastroesophageal varices and variceal hemorrhage in cirrhosis. Am J Gastroenterol 2007; 102: 2086-2102

14 Hwang JH, Shergill AK, Acosta RD et al. The role of endoscopy in the management of variceal hemorrhage. Gastrointest Endosc 2014; 80: $221-227$

15 Moher D, Liberati A, Tetzlaff J et al. Preferred reporting items for systematic reviews and meta-analyses: the PRISMA statement. Ann Intern Med 2009; 151: 264-269

16 Stang A. Critical evaluation of the Newcastle-Ottawa scale for the assessment of the quality of nonrandomized studies in meta-analyses. Eur J Epidemiol 2010; 25: 603-605

17 Berner JS, Gaing AA, Sharma $R$ et al. Sequelae after esophageal variceal ligation and sclerotherapy: a prospective randomized study. Am J Gastroenterol 1994; 89: 852-858

18 Kulkarni SG, Parikh SS, Dhawan PS et al. High frequency of bacteremia with endoscopic treatment of esophageal varices in advanced cirrhosis. Indian J Gastroenterol 1999; 18: 143 - 145

19 Bonilha DQ Correia LM, Monaghan M et al. Prospective study of bacteremia rate after elective band ligation and sclerotherapy with cyanoacrylate for esophageal varices in patients with advanced liver disease. Arq Gastroenterol 2011; 48: 248-251

20 Cohen LB, Korsten MA, Scherl EJ et al. Bacteremia after endoscopic injection sclerosis. Gastrointest Endosc 1983; 29: 198-200

21 Brayko CM, Kozarek RA, Sanowski RA et al. Bacteremia during esophageal variceal sclerotherapy: its cause and prevention. Gastrointest Endosc 1985; 31: 10-12

22 Snady H, Korsten MA, Waye JD. The relationship of bacteremia to the length of injection needle in endoscopic variceal sclerotherapy. Gastrointest Endosc 1985; 31: $243-246$

23 Low DE, Shoenut JP, Kennedy JK et al. Infectious complications of endoscopic injection sclerotherapy. Arch Intern Med 1986; 146: 569 - 571 
24 Hegnhoj J, Andersen JR, Jarlov JO et al. Bacteriaemia after injection sclerotherapy of oesophageal varices. Liver 1988; 8: 167-171

25 Lorgat F, Madden MV, Kew $G$ et al. Bacteremia after injection of esophageal varices. Surg Endosc 1990; 4: 18-19

26 Rolando N, Gimson A, Philpott-Howard J et al. Infectious sequelae after endoscopic sclerotherapy of oesophageal varices: role of antibiotic prophylaxis. J Hepatol 1993; 18: 290-294

27 Selby WS, Norton ID, Pokorny CS et al. Bacteremia and bacterascites after endoscopic sclerotherapy for bleeding esophageal varices and prevention by intravenous cefotaxime: a randomized trial. Gastrointest Endosc 1994; 40: 680 - 684

28 da Silveira RohrMR, Siqueira ES, Brant CQ et al. Prospective study of bacteremia rate after elastic band ligation and sclerotherapy of esophageal varices in patients with hepatosplenic schistosomiasis. Gastrointest Endosc 1997; 46: 321 - 323

29 Maulaz EB, de Mattos AA, Pereira-Lima J et al. Bacteremia in cirrhotic patients submitted to endoscopic band ligation of esophageal varices. Arq Gastroenterol 2003; 40: 166-172

30 Rey JR, Axon A, Budzynska A et al. Guidelines of the European Society of Gastrointestinal Endoscopy (E.S.G.E.) antibiotic prophylaxis for gastrointestinal endoscopy. European Society of Gastrointestinal Endoscopy. Endoscopy 1998; 30: 318-324

31 Laine L, Cook D. Endoscopic ligation compared with sclerotherapy for treatment of esophageal variceal bleeding. A meta-analysis. Ann Intern Med 1995; 123: 280-287

32 Woods KL, Qureshi WA. Long-term endoscopic management of variceal bleeding. Gastrointest Endosc Clin N Am 1999; 9: 253 - 270
33 Stiegmann GV, Goff JS, Michaletz-Onody PA et al. Endoscopic sclerotherapy as compared with endoscopic ligation for bleeding esophageal varices. NEJM 1992; 326: $1527-1532$

34 Laine L, el-Newihi HM, Migikovsky B et al. Endoscopic ligation compared with sclerotherapy for the treatment of bleeding esophageal varices. Ann Intern Med 1993; 119: 1 - 7

35 Baroncini D, Milandri GL, Borioni $D$ et al. A prospective randomized trial of sclerotherapy versus ligation in the elective treatment of bleeding esophageal varices. Endoscopy 1997; 29: 235-240

36 Arakawa M, Masuzaki T, Okuda K. Pathology of fundic varices of the stomach and rupture. J Gastroenterol Hepatol 2002; 17: 1064-1069

37 Wahl P, Lammer F, Conen D et al. Septic complications after injection of N-butyl-2-cyanoacrylate: report of two cases and review. Gastrointest Endosc 2004; 59: $911-916$

38 Rogers KL, Fey PD, Rupp ME. Coagulase-negative staphylococcal infections. Infect Dis Clin North Am 2009; 23: 73-98

39 Chang YJ, Park JJ, Joo MK et al. Long-term outcomes of prophylactic endoscopic histoacryl injection for gastric varices with a high risk of bleeding. Dig Dis Sci 2010; 55: $2391-2397$

40 Berg RD. Bacterial translocation from the gastrointestinal tract. Adv Exp Med Biol 1999; 473: 11-30

41 Garcia-Tsao G. Bacterial translocation: cause or consequence of decompensation in cirrhosis? J Hepatol 2001; 34: 150-155

42 Lee S, Son SC, Han MJ et al. Increased intestinal macromolecular permeability and urine nitrite excretion associated with liver cirrhosis with ascites. World J Gastroenterol 2008; 14: 3884-3890

$43 \mathrm{Fukui} \mathrm{H}$. How leaky gut and endotoxemia induce bacterial infection in cirrhosis and gastrointestinal hemorrhage? J Gastroenterol Hepatol 2011; 26 : $423-425$

\section{Supplemental Tables 3, 4}

supplementary content viewable at:

http://dx.doi.org/10.1055/s-0034-1392552 